\title{
Sur la longueur de la fraction continue de $\alpha^{n}$
}

\author{
par \\ Guillaume Grisel (Caen)
}

I. Introduction. Soit $x$ un nombre rationnel. Notons $d(x)$ le nombre de termes de la fraction continue de longueur paire de $x$. Y. Pourchet dans une lettre à M. Mendès France d'une part et G. Choquet dans une série de comptes rendus à l'Académie des Sciences [2] d'autre part, ont démontré que si $x$ n'est ni un entier ni l'inverse d'un entier, alors $\sup d\left(x^{n}\right)=\infty$. La démonstration de Y. Pourchet, non publiée, est résumée dans l'article de A. J. van der Poorten [7].

Nous nous intéressons à un problème similaire portant sur les nombres quadratiques réels. Si $\alpha$ est un nombre quadratique réel, son développement en fraction continue est périodique, de longueur de période primitive $l(\alpha)$. En Novembre 1992, lors d'un congrés à Tokyo, M. Mendès France posait la question suivante ([5], problème $\left.\mathrm{n}^{\circ} 6\right)$ : pour tout nombre quadratique réel $\alpha$, est-il vrai que

$$
\limsup _{n \rightarrow \infty} l\left(\alpha^{n}\right)=\infty ?
$$

Une première réponse, à mettre en parallèle avec le cas des entiers dans le problème sur les nombres rationnels, peut déjà être faite : R. Paysant-Le Roux et E. Dubois remarquent dans [6] que si $\alpha$ est une unité quadratique, alors $l(\alpha) \leq 2$. La question doit donc être reformulée en ne considérant plus que les nombres quadratiques réels qui ne sont pas des unités quadratiques. L'existence du nombre $l\left(\alpha^{n}\right)$ pour tout $n \geq 1$ est équivalente à $\alpha^{2} \notin \mathbb{Q}$, il est donc bien entendu que si ce n'est pas le cas, $n$ désignera un nombre impair. Nous montrons que, pour une large classe de nombres réels quadratiques, la réponse à la question de M. Mendès France est non seulement positive mais que $l\left(\alpha^{n}\right)$ tend vers l'infini avec $n$ et ceci de manière explicite. Nous établissons :

ThÉorème. Soit $\alpha=(a+b \sqrt{d}) / c, a \in \mathbb{Z}, b \in \mathbb{Z}^{*}, c \in \mathbb{N}^{*}, \operatorname{pgcd}(a, b, c)=$ 1 et $d \geq 2$ sans facteur carré. Posons $f_{2}=\operatorname{pgcd}\left(a^{2}+b^{2} d, 2 a b, c^{2}\right), c_{2}=$ $c^{2} / f_{2}$ et $N_{2}=\left(a^{2}-b^{2} d\right)^{2} / f_{2}^{2}$. Supposons que $\alpha$ vérifie l'une des conditions suivantes : 
(i) $N_{2} \not \equiv 0\left(\bmod c_{2}\right)$,

(ii) $\operatorname{pgcd}(a, b)>1$,

(iii) $\operatorname{pgcd}(a, d)>1$,

(iv) $a^{2}-b^{2} d$ pair et c impair.

Alors il existe une constante effectivement calculable $K$ strictement positive et indépendante de $n$, telle que pour tout $n \geq 2$,

$$
l\left(\alpha^{n}\right)>K \frac{2^{n}}{n} .
$$

La preuve de ce théorème consiste en la détermination, pour tout $n \geq 1$, d'un générateur de $O_{n}$ l'anneau des stabilisateurs du module $\mathbb{Z}+\mathbb{Z} \alpha^{n}$, en fonction de conditions sur $\alpha$. Plus précisément, il s'agit de faire apparaître des grandes puissances d'entiers dans le conducteur de $O_{n}$. Deux minorations, l'une de $l\left(\alpha^{n}\right)$ (proposition 5) due à E. P. Golubeva [4] et faisant intervenir l'unité fondamentale et le discriminant de $O_{n}$, et l'autre de l'indice du groupe des unités de $O_{n}$ dans le groupe des unités du corps $\mathbb{Q}(\alpha)$ (proposition 6), reposant sur un lemme de $\mathrm{H}$. Cohen [3], sont à la base de cette démonstration.

II. La forme canonique de $\alpha^{n}$. Considérons $\alpha=(a+b \sqrt{d}) / c$, où $a \in \mathbb{Z}, b \in \mathbb{Z}^{*}, c \in \mathbb{N}^{*}, \operatorname{pgcd}(a, b, c)=1$ et $d \geq 2$ sans facteur carré, et posons $N=a^{2}-b^{2} d$. Notons, pour tout $n \geq 1$,

$$
\alpha^{n}=\left(\frac{a+b \sqrt{d}}{c}\right)^{n}=\frac{a_{n}+b_{n} \sqrt{d}}{c_{n}}, \quad \operatorname{avec} \operatorname{pgcd}\left(a_{n}, b_{n}, c_{n}\right)=1 \text { et } c_{n}>0 .
$$

Définissons, pour tout $n \geq 1$, les entiers $a_{n}^{\prime}$ et $b_{n}^{\prime} \operatorname{par}(a+b \sqrt{d})^{n}=a_{n}^{\prime}+b_{n}^{\prime} \sqrt{d}$, et posons $f_{n}=\operatorname{pgcd}\left(a_{n}^{\prime}, b_{n}^{\prime}, c^{n}\right)$.

Proposition 1. Pour tout $n \geq 1, f_{n}=2^{l_{n}^{\prime}}(\operatorname{pgcd}(a, d, c))^{l_{n}}$, avec :

(i) $l_{n}=[n / 2]$;

(ii) Si l'un des nombres $a^{2}-b^{2} d$ ou c est impair, alors $l_{n}^{\prime}=0$;

(iii) Si les deux nombres $a^{2}-b^{2} d$ et $c$ sont tous les deux pairs, alors

$$
l_{n}^{\prime}= \begin{cases}0 & \text { si } d \equiv 2(\bmod 4), \\ {[n / 2]} & \text { si } d \equiv 3(\bmod 4), \\ n-1 & \text { si } d \equiv 1(\bmod 8), \\ n-1 & \text { si } d \equiv 5(\bmod 8) \text { et si } 3 \text { ne divise pas } n, \\ n & \text { si } d \equiv 5(\bmod 8) \text { et si } 3 \text { divise } n .\end{cases}
$$

Démonstration. Le cas $a=0$ est trivial : $\operatorname{comme} \operatorname{pgcd}(b, c)=1$ et $d$ est sans facteur carré on a alors $f_{2 n+1}=\operatorname{pgcd}\left(b^{2 n+1} d^{n}, c^{2 n+1}\right)=$ $(\operatorname{pgcd}(c, d))^{n}$. Nous supposons donc $a \neq 0$ dans la suite de la démonstration.

Posons, pour tout $n \geq 1, \delta_{n}=\operatorname{pgcd}\left(a_{n}^{\prime}, b_{n}^{\prime}\right)$. Nous explicitons dans un premier temps les diviseurs premiers de $\delta_{n}$, puis nous déterminons les puis- 
sances avec lesquelles ils divisent $\delta_{n}$. Nous en déduisons alors $f_{n}$. Les facteurs communs à $a$ et $b$ divisent $\delta_{n}$, mais ne divisent pas $f_{n} \operatorname{car} \operatorname{pgcd}(a, b, c)=1$. On peut donc supposer, pour l'étude de $f_{n}, \delta_{1}=\operatorname{pgcd}(a, b)=1$.

Lemme 1. Supposons $\delta_{1}=1$. Pour tout $n \geq 1$, si p premier divise $\delta_{n}$ alors $p=2$ ou $p$ divise $\operatorname{pgcd}(a, d)$.

Démonstration. On a, pour tout $n \geq 1$,

$$
a_{n+1}^{\prime}=a_{n}^{\prime} a+b_{n}^{\prime} b d, \quad b_{n+1}^{\prime}=a_{n}^{\prime} b+b_{n}^{\prime} a .
$$

Donc si $p$ divise $\delta_{n}$, il divise aussi $\delta_{n+1}$. Alors quitte à changer $n$ en $n+1$, on peut supposer $n$ pair. En posant $n=2 m$, on a alors

$$
a_{n}^{\prime}=a_{m}^{\prime 2}+b_{m}^{\prime 2} d, \quad b_{n}^{\prime}=2 a_{m}^{\prime} b_{m}^{\prime} .
$$

On en déduit que ou $p=2$, ou $p$ divise $\delta_{m}$, ou $p$ divise $\operatorname{pgcd}\left(a_{m}^{\prime}, d\right)$.

Si $m=1$, comme on a supposé $\operatorname{pgcd}(a, b)=1$, alors $p=2$ ou $p$ divise $\operatorname{pgcd}\left(a_{1}^{\prime}, d\right)=\operatorname{pgcd}(a, d)$.

Si $m>1$, alors ou $p=2$, et c'est fini, ou $p$ divise $\operatorname{pgcd}\left(a_{m}^{\prime}, d\right)$, ou $p$ divise $\delta_{m}$.

(a) Si $p$ divise $\operatorname{pgcd}\left(a_{m}^{\prime}, d\right)$, alors par (1), il divise $\operatorname{pgcd}(a, d)$ ou il divise $\operatorname{pgcd}\left(a_{m-1}^{\prime}, d\right)$ et on obtient par récurrence descendante jusqu'à $m=1, p$ divise $\operatorname{pgcd}(a, d)$.

(b) Si $p$ divise $\delta_{m}$, alors soit $[(m+1) / 2]=1$, soit $[(m+1) / 2]>1$.

Dans ce second cas, on a alors ou $p=2$ et c'est fini, ou $p$ divise $\operatorname{pgcd}\left(a_{[(m+1) / 2]}^{\prime}, d\right)$ et on effectue $(\mathrm{a})$, ou $p$ divise $\delta_{[(m+1) / 2]}$ et on recommence (b).

On s'arrête donc en entrant dans (a), ou lorsque $[(m+1) / 2]=1$. Il suit donc $p=2$ ou $p$ divise $\operatorname{pgcd}(a, d)$.

Comme $d$ est sans facteur carré, on peut écrire $\operatorname{pgcd}(a, d, c)=p_{1} \ldots p_{s}$ où les $p_{i}$ sont premiers et tous distincts. Or il est facile de voir, en développant $(a+b \sqrt{d})^{n}$ par la formule du binôme, que pour tout $n \geq 2$, le $\operatorname{pgcd}(a, d, c)$ divise $\delta_{n}$, et que par conséquent il divise $f_{n}$. On en déduit donc par le lemme 1 ,

$$
f_{n}=2^{l_{n}^{\prime}} p_{1}^{l_{1, n}} \ldots p_{s}^{l_{s, n}}, \quad l_{i, n} \geq 1 .
$$

- Montrons le point (i) de la proposition 1. Pour tout $i$ compris entre 1 et $s$, comme par hypothèse $\operatorname{pgcd}(a, b, c)=1, p_{i}$ ne divise pas $b$, et puisque $d$ est sans facteur carré, $p_{i}$ divise exactement $N=a^{2}-b^{2} d$. D'où, si $v_{p_{i}}(x)$ désigne la valuation $p_{i}$-adique de l'entier $x, v_{p_{i}}\left(N^{n}\right)=v_{p_{i}}\left(a_{n}^{\prime 2}-b_{n}^{\prime 2} d\right)=n$. On a alors :

- soit $v_{p_{i}}\left(a_{n}^{\prime}\right) \leq v_{p_{i}}\left(b_{n}^{\prime}\right)$, et alors $v_{p_{i}}\left(N^{n}\right)=v_{p_{i}}\left(a_{n}^{\prime 2}\right)$, d'où $n$ est pair et $v_{p_{i}}\left(\delta_{n}\right)=v_{p_{i}}\left(a_{n}^{\prime}\right)=n / 2$

- soit $v_{p_{i}}\left(b_{n}^{\prime}\right)<v_{p_{i}}\left(a_{n}^{\prime}\right)$, et alors $v_{p_{i}}\left(N^{n}\right)=v_{p_{i}}\left(b_{n}^{\prime 2} d\right)=n$; il suit que $n$ est impair et $v_{p_{i}}\left(\delta_{n}\right)=v_{p_{i}}\left(b_{n}^{\prime}\right)=(n-1) / 2$. 
Comme $v_{p_{i}}\left(c^{n}\right) \geq n$, on obtient donc $l_{i, n}=l_{n}=[n / 2]$.

- Le point (ii) de la proposition 1 est immédiat.

On déduit le point (iii) de la proposition 1 à partir de la valuation 2adique de $\delta_{n}$, qui fait l'objet du lemme suivant :

Lemme 2. Si $v_{2}\left(\delta_{1}\right)=0$ et $N=a^{2}-b^{2} d$ pair, alors pour tout $n \geq 1$,

$$
v_{2}\left(\delta_{n}\right)= \begin{cases}{[n / 2]} & \text { si } d \neq \equiv 1(\bmod 4), \\ n-1 & \text { si } d \equiv 1(\bmod 8), \\ n-1 & \text { si } d \equiv 5(\bmod 8) \text { et si } 3 \text { ne divise pas } n, \\ n & \text { si } d \equiv 5(\bmod 8) \text { et si } 3 \text { divise } n\end{cases}
$$

Démonstration. Si $d \equiv 2(\bmod 4)$ et $N=a^{2}-b^{2} d$ pair, alors $\operatorname{pgcd}(a, d)$ est pair. Il existe donc un indice $i$ tel que $p_{i}=2$, et d'après la démonstration du point (i) de la proposition $1, v_{2}\left(\delta_{n}\right)=l_{n}=[n / 2]$.

Si $d \equiv 3(\bmod 4)$, comme $\delta_{1}=1$ et $N$ pair, alors $v_{2}(N)=1$. En reprenant la démonstration du point (i) avec $v_{2}\left(N^{n}\right)$, on obtient pour tout $n \geq 1, v_{2}\left(\delta_{n}\right)=[n / 2]$.

Partant de (1), on peut écrire, pour tout $n \geq 1$,

$$
a_{n+2}^{\prime}=2 a a_{n+1}^{\prime}-N a_{n}^{\prime}, \quad b_{n+2}^{\prime}=2 a b_{n+1}^{\prime}-N b_{n}^{\prime} .
$$

Comme $\delta_{1}=1$, de $N$ pair et $d \equiv 1(\bmod 4)$, on a $v_{2}\left(a_{1}^{\prime}\right)=v_{2}\left(b_{1}^{\prime}\right)=0$ et $v_{2}\left(a_{2}^{\prime}\right)=v_{2}\left(b_{2}^{\prime}\right)=1$. On raisonne alors par récurrence sur $n$.

Si $d \equiv 1(\bmod 8)$, alors $v_{2}(N) \geq 3$. Supposons que $v_{2}\left(a_{n}^{\prime}\right)=v_{2}\left(b_{n}^{\prime}\right)=n-$ 1 et $v_{2}\left(a_{n+1}^{\prime}\right)=v_{2}\left(b_{n+1}^{\prime}\right)=n$, et montrons que $v_{2}\left(a_{n+2}^{\prime}\right)=v_{2}\left(b_{n+2}^{\prime}\right)=n+1$.

Par $(2)$, on a $v_{2}\left(a_{n+2}^{\prime}\right)=\min \left\{v_{2}\left(2 a a_{n+1}^{\prime}\right), v_{2}\left(N a_{n}^{\prime}\right)\right\}=\min \{n+1, n+$ $2+k\}$ avec $k=v_{2}(N)-3$. D'où $v_{2}\left(a_{n+2}^{\prime}\right)=n+1$. De la même manière, on obtient $v_{2}\left(b_{n+2}^{\prime}\right)=n+1$.

Si $d \equiv 5(\bmod 8)$, alors $v_{2}(N)=2$. Supposons que $v_{2}\left(a_{3 k+1}^{\prime}\right)=v_{2}\left(b_{3 k+1}^{\prime}\right)$ $=3 k$ et $v_{2}\left(a_{3 k+2}^{\prime}\right)=v_{2}\left(b_{3 k+2}^{\prime}\right)=3 k+1$. Montrons alors que $v_{2}\left(\delta_{3(k+1)}\right)=$ $3(k+1), v_{2}\left(a_{3(k+1)+1}^{\prime}\right)=v_{2}\left(b_{3(k+1)+1}^{\prime}\right)=3(k+1)$ et $v_{2}\left(a_{3(k+1)+2}^{\prime}\right)=$ $v_{2}\left(b_{3(k+1)+2}^{\prime}\right)=3(k+1)+1$.

On a

$$
v_{2}\left(\frac{2 a a_{3 k+2}^{\prime}}{2^{3 k+2}}-\frac{N a_{3 k+1}^{\prime}}{2^{3 k+2}}\right) \geq 1,
$$

et par $(2), v_{2}\left(a_{3(k+1)}^{\prime}\right) \geq 3(k+1)$. Avec les mêmes arguments, on déduit $v_{2}\left(b_{3(k+1)}^{\prime}\right) \geq 3(k+1)$, et donc $v_{2}\left(\delta_{3(k+1)}\right) \geq 3(k+1)$. En utilisant de nouveau les inégalités $(2)$, on obtient

$$
v_{2}\left(a_{3(k+1)+1}^{\prime}\right)=v_{2}\left(b_{3(k+1)+1}^{\prime}\right)=v_{2}\left(\delta_{3(k+1)}\right)=3(k+1) .
$$

Or, d'après (1), si un entier divise $\delta_{n}$ alors il divise $\delta_{n+1}$. Il en suit donc $v_{2}\left(\delta_{3(k+1)}\right)=3(k+1)$. On obtient finalement, par $(2), v_{2}\left(a_{3(k+1)+2}^{\prime}\right)=$ $v_{2}\left(b_{3(k+1)+2}^{\prime}\right)=v_{2}\left(\delta_{3(k+1)+2}\right)=3(k+1)+1$. 
- Montrons le point (iii) de la proposition 1. Si $N$ et $c$ sont pairs, alors $v_{2}\left(f_{n}\right)=\min \left\{v_{2}\left(\delta_{n}\right), v_{2}\left(c^{n}\right)\right\}$. Or d'après le lemme $2, v_{2}\left(f_{n}\right) \leq n \leq v_{2}\left(c^{n}\right)$.

Si $d \equiv 2(\bmod 4)$, comme $\operatorname{pgcd}(a, b)=1$ et $d$ est sans facteur carré, alors $v_{2}(\operatorname{pgcd}(a, d, c))=1$. Or, pour tout $n \geq 1$, on a, $f_{n}=2^{l_{n}^{\prime}}(\operatorname{pgcd}(a, d, c))^{[n / 2]}$ et d'après le lemme $2, v_{2}\left(f_{n}\right)=[n / 2]$. Il suit donc $l_{n}^{\prime}=0$.

Si $d \not \equiv 2(\bmod 4)$, alors $\operatorname{pgcd}(a, d, c)$ est impair, et donc pour tout $n \geq 1$, $l_{n}^{\prime}=v_{2}\left(\delta_{n}\right)$ donné par le lemme 2 , ce qui termine la démonstration de la proposition 1.

III. Etude de l'anneau des stabilisateurs. Pour tout $n \geq 1$, soit $O_{n}$ l'anneau des stabilisateurs du module $\mathbb{Z}+\mathbb{Z} \alpha^{n}$, i.e. l'ensemble des $\gamma \in \mathbb{Q}(\sqrt{d})$ tels que $\gamma\left(\mathbb{Z}+\mathbb{Z} \alpha^{n}\right) \subset \mathbb{Z}+\mathbb{Z} \alpha^{n}$. Nous nous proposons dans ce paragraphe de donner, au travers de trois propositions, une description de $O_{n}$ en fonction de $\alpha$.

Considérons, pour tout $n \geq 1$, l'équation minimale de $\alpha^{n}$ :

$$
\omega_{1}(n) X^{2}+\omega_{2}(n) X+\omega_{3}(n)=0,
$$

$\operatorname{avec}\left(\omega_{1}(n), \omega_{2}(n), \omega_{3}(n)\right) \in \mathbb{Z}^{3}, \omega_{1}(n)>0$ et $\operatorname{pgcd}\left(\omega_{1}(n), \omega_{2}(n), \omega_{3}(n)\right)=1$. On peut écrire, en reprenant les notations du paragraphe II,

$$
\frac{\omega_{3}(n)}{\omega_{1}(n)}=\frac{a_{n}^{2}-b_{n}^{2} d}{c_{n}^{2}} \quad \text { et } \quad \frac{\omega_{2}(n)}{\omega_{1}(n)}=\frac{2 a_{n}}{c_{n}} .
$$

En posant $\gamma_{n}=\operatorname{pgcd}\left(2 a_{n} c_{n}, a_{n}^{2}-b_{n}^{2} d, c_{n}^{2}\right)$, il suit alors

$$
\omega_{1}(n)=c_{n}^{2} / \gamma_{n} \text {. }
$$

Remarquons que de manière plus simple, on a $\gamma_{n}=\operatorname{pgcd}\left(2 c_{n}, a_{n}^{2}-b_{n}^{2} d, c_{n}^{2}\right)$. En effet, $\operatorname{pgcd}\left(2 c_{n}, a_{n}^{2}-b_{n}^{2} d, c_{n}^{2}\right)$ divise $\gamma_{n}$. Réciproquement, tous les diviseurs de $\gamma_{n}$ divisent $\operatorname{pgcd}\left(2 c_{n}, a_{n}^{2}-b_{n}^{2} d, c_{n}^{2}\right)$ sauf peut être s'ils divisent $a_{n}$. Or, si un premier $p$ divise $a_{n}$ et $\gamma_{n}$, il divise $b_{n}^{2} d$. Il ne divise pas $b_{n}$, car $\operatorname{pgcd}\left(a_{n}, b_{n}, c_{n}\right)=1$ et par suite il divise $d$. Or comme $d$ est sans facteur carré, il divise exactement $a_{n}^{2}-b_{n}^{2} d$ et par conséquent, il divise exactement $\gamma_{n}$. Mais alors, comme il divise $c_{n}^{2}$, il divise aussi $\operatorname{pgcd}\left(2 c_{n}, a_{n}^{2}-b_{n}^{2} d, c_{n}^{2}\right)$.

D'après Z. I. Borevitch et I. R. Chafarevitch [1], p. 152, on a

$$
O_{n}=\mathbb{Z}\left[\omega_{1}(n) \alpha^{n}\right]=\mathbb{Z}+\mathbb{Z} \omega_{1}(n) \alpha^{n} .
$$

Pour tout $n \geq 1$, posons $N_{n}=a_{n}^{2}-b_{n}^{2} d$. En considérant les trois cas suivants : $c_{n}$ ne divise pas $N_{n}, c_{n}$ divise $N_{n}$ mais $c_{n}^{2}$ ne divise pas $N_{n}$, et $c_{n}^{2}$ divise $N_{n}$, on détermine alors $c_{n}^{2} / \gamma_{n}$ et donc $O_{n}$ en fonction de $a_{n}, b_{n}, c_{n}$ et $d$. On obtient :

Proposition 2. Pour tout $n \geq 1$, l'ordre $O_{n}$ est égal à $\mathbb{Z}\left[\xi_{n}\right]$, où l'entier quadratique $\xi_{n}$ est donné par le tableau suivant: 
Tableau 1

\begin{tabular}{|c|c|c|c|}
\hline & $N_{n} \equiv 0\left(\bmod c_{n}^{2}\right)$ & $\begin{array}{c}N_{n} \equiv 0\left(\bmod c_{n}\right) \\
\text { et } \\
N_{n} \not \equiv 0\left(\bmod c_{n}^{2}\right)\end{array}$ & $N_{n} \not \equiv 0\left(\bmod c_{n}\right)$ \\
\hline $\begin{array}{c}d \equiv 2,3(\bmod 4), \text { ou } \\
d \equiv 1(\bmod 4) \text { et } c_{n} \text { impair }\end{array}$ & $\xi_{n}=k_{n} \sqrt{d}$ & $\xi_{n}=k_{n} \sqrt{d}$ & $\xi_{n}=k_{n} \sqrt{d}$ \\
\hline $\begin{array}{c}\text { et } \\
c_{n} \text { pair }\end{array}$ & $\xi_{n}=\frac{1+k_{n} \sqrt{d}}{2}$ & $\begin{array}{c}\xi_{n}=\left(1+k_{n} \sqrt{d}\right) / 2 \\
\text { si } v_{2}\left(N_{n}\right)>v_{2}\left(c_{n}\right)\end{array}$ & $\begin{array}{c}\xi_{n}=\left(1+k_{n} \sqrt{d}\right) / 2 \\
\text { si } v_{2}\left(N_{n}\right)>v_{2}\left(c_{n}\right)\end{array}$ \\
\cline { 3 - 4 } & $\begin{array}{c}\xi_{n}=k_{n} \sqrt{d} \\
\text { si } v_{2}\left(N_{n}\right)=v_{2}\left(c_{n}\right)\end{array}$ & $\begin{array}{c}\xi_{n}=k_{n} \sqrt{d} \\
\text { si } v_{2}\left(N_{n}\right) \leq v_{2}\left(c_{n}\right)\end{array}$ \\
\hline
\end{tabular}

avec $k_{n}$ entier vérifiant :

(i) $k_{n}= \begin{cases}\left|c_{n} b_{n} / \gamma_{n}\right| & \text { si } O_{n} \subset \mathbb{Z}[\sqrt{d}], \\ \left|2 c_{n} b_{n} / \gamma_{n}\right| & \text { si } O_{n} \not \subset \mathbb{Z}[\sqrt{d}]\end{cases}$

(ii) $k_{n}>\left|b_{n}\right|$ si et seulement si $N_{n} \not \equiv 0\left(\bmod c_{n}\right)$.

La divisibilité de $N_{n}$ par $c_{n}$ joue un rôle important dans la forme de $\xi_{n}$. Nous allons en déterminer des conditions équivalentes.

Proposition 3. Les assertions suivantes sont équivalentes :

(i) Il existe $n \geq 2$ tel que $c_{n}$ divise $N_{n}$.

(ii) c divise $N, \operatorname{pgcd}(a, d, c)=1,0<v_{2}(c) \leq v_{2}(N)-1$ ou $v_{2}(c)=0$.

(iii) Pour tout $n \geq 1, c_{n}$ divise $N_{n}$.

(iv) $c_{2}$ divise $N_{2}$.

Dé monstration. (iii) $\Rightarrow$ (iv) et (iv) $\Rightarrow$ (i) sont claires. Nous allons montrer que (i) $\Rightarrow$ (ii) et que (ii) $\Rightarrow$ (iii).

Pour tout $n \geq 1$, on a $c_{n}=c^{n} / f_{n}$ et $N_{n}=N^{n} / f_{n}^{2}$, où $f_{n}$ est le pgcd défini au paragraphe II. On peut donc écrire

$$
\left(c_{n} \text { divise } N_{n}\right) \Leftrightarrow\left(c^{n} \text { divise } N^{n} / f_{n}\right) \text {. }
$$

D'après la proposition $1, f_{n}$ est de la forme $f_{n}=2^{l^{\prime}}(\operatorname{pgcd}(a, d, c))^{l_{n}}$. Posons comme précédemment $\operatorname{pgcd}(a, d, c)=p_{1} \ldots p_{s}$ avec les $p_{i}$ premiers et tous distincts. Alors $c_{n}$ divise $N_{n}$ si et seulement si les trois conditions suivantes sont vérifiées :

1) $c$ divise $N$;

2) pour tout $i=1, \ldots, s, v_{p_{i}}\left(c^{n}\right) \leq v_{p_{i}}\left(N^{n}\right)-v_{p_{i}}\left(f_{n}\right)$;

3) $v_{2}\left(c^{n}\right) \leq v_{2}\left(N^{n}\right)-v_{2}\left(f_{n}\right)$.

(i) $\Rightarrow$ (ii). D'après 1$), c$ divise $N$. Déduisons de 2) que $\operatorname{pgcd}(a, d, c)=1$. Raisonnons par l'absurde, et supposons que $\operatorname{pgcd}(a, d, c) \neq 1$. D'après la proposition 1 , on a $l_{n}=[n / 2]$. Donc, il existe un indice $i, 1 \leq i \leq s$, 
tel que $v_{p_{i}}\left(f_{n}\right)=[n / 2] \geq 1$, car $n \geq 2$. De plus, comme par hypothèse $\operatorname{pgcd}(a, b, c)=1, p_{i}$ ne divise pas $b$. Il suit alors que $v_{p_{i}}(N)=v_{p_{i}}(d)=1$, car $d$ est sans facteur carré. De 2) on obtient donc $n v_{p_{i}}(c) \leq n-1$, d'où $v_{p_{i}}(c)=0$, ce qui est une contradiction.

Reste à montrer $0<v_{2}(c)<v_{2}(N)$ ou $v_{2}(c)=0$. Si $v_{2}\left(f_{n}\right) \geq 1$, alors $v_{2}(c)>0$, et la condition 3$)$ implique

$$
0<v_{2}(c) \leq v_{2}(N)-v_{2}\left(f_{n}\right) / n \leq v_{2}(N)-1 / n<v_{2}(N) .
$$

Si $\left.v_{2}\left(f_{n}\right)=0,3\right)$ devient $v_{2}(c) \leq v_{2}(N)$, et d'après la proposition 1 , on a $v_{2}(c)=0$ ou $v_{2}(N)=0$.

(ii) $\Rightarrow$ (iii). Si (ii) est vraie alors, pour tout $n \geq 1,1$ ) et 2 ) sont vérifiées. Si $v_{2}(c)=0$ alors pour tout $n \geq 1, v_{2}\left(f_{n}\right)=0$ et 3$)$ est vérifiée. Si $0<$ $v_{2}(c)<v_{2}(N)$, comme d'après la proposition $1, v_{2}\left(f_{n}\right) \leq n$, on a alors $0<v_{2}(c) \leq v_{2}(N)-1 \leq v_{2}(N)-v_{2}\left(f_{n}\right) / n$ et 3$)$ est vérifiée.

Nous déterminons maintenant la forme de $\xi_{n}$ en fonction de conditions sur $\alpha$.

Proposition 4. (i) Si $d \not \equiv 1(\bmod 4)$, alors pour tout $n \geq 1, \xi_{n}=k_{n} \sqrt{d}$.

(ii) Si $d \equiv 1(\bmod 4)$ et $\xi_{1}=k_{1} \sqrt{d}$, alors pour tout $n \geq 1, \xi_{n}=k_{n} \sqrt{d}$.

(iii) Si $d \equiv 1(\bmod 8)$ et $\xi_{1}=\left(1+k_{1} \sqrt{d}\right) / 2$, alors pour tout $n \geq 1$, $\xi_{n}=\left(1+k_{n} \sqrt{d}\right) / 2$.

(iv) Si $d \equiv 5(\bmod 8)$, et $\xi_{1}=\left(1+k_{1} \sqrt{d}\right) / 2$, alors

$$
\xi_{n}= \begin{cases}k_{n} \sqrt{d} & \text { si } 3 \text { divise } n \\ \left(1+k_{n} \sqrt{d}\right) / 2 & \text { si } 3 \text { ne divise pas } n .\end{cases}
$$

Démonstration. (i) Si $d \not \equiv 1(\bmod 4)$, alors pour tout $n \geq 1$, l'anneau $O_{n}$ est inclus dans $\mathbb{Z}[\sqrt{d}]$.

(ii) Si $d \equiv 1(\bmod 4)$ et $\xi_{1}=k_{1} \sqrt{d}$, alors d'après le tableau 1 , on a $v_{2}(c)=0$ ou $v_{2}(N) \leq v_{2}(c)$.

Si $v_{2}(c)=0$, alors pour tout $n \geq 1, v_{2}\left(c_{n}\right)=n v_{2}(c)-v_{2}\left(f_{n}\right)=0$, et grâce au tableau $1, \xi_{n}=k_{n} \sqrt{d}$.

Si $v_{2}(N) \leq v_{2}(c)$, on peut écrire, pour tout $n \geq 1$,

$$
v_{2}(N) \leq v_{2}(c)+v_{2}\left(f_{n}\right) / n,
$$

c'est-à-dire $v_{2}\left(N_{n}\right) \leq v_{2}\left(c_{n}\right)$, et d'après le tableau $1, \xi_{n}=k_{n} \sqrt{d}$.

(iii) De $\xi_{1}=\left(1+k_{1} \sqrt{d}\right) / 2$ il suit, d'après le tableau $1, v_{2}(N)>v_{2}(c)>0$. Si $d \equiv 1(\bmod 8)$, on en déduit alors, par la proposition 1 , que pour tout $n \geq 1, v_{2}\left(f_{n}\right)=n-1$, et on peut écrire

$$
0<v_{2}(c)<v_{2}(N)-v_{2}\left(f_{n}\right) / n,
$$

qui équivaut à $v_{2}\left(c_{n}\right)<v_{2}\left(N_{n}\right)$. D'où, grâce au tableau $1, \xi_{n}=\left(1+k_{n} \sqrt{d}\right) / 2$. 
(iv) De $\xi_{1}=\left(1+k_{1} \sqrt{d}\right) / 2$ on a, comme précédemment, $v_{2}(N)>v_{2}(c)>$ 0 . Si $d \equiv 5(\bmod 8)$, il suit alors par la proposition 1 , pour tout $n \geq 1$,

$$
v_{2}\left(f_{n}\right)= \begin{cases}n & \text { si } 3 \text { divise } n, \\ n-1 & \text { si } 3 \text { ne divise pas } n .\end{cases}
$$

Si 3 ne divise pas $n$, en reprenant la démonstration du point (iii), on a $\xi_{n}=\left(1+k_{n} \sqrt{d}\right) / 2$.

Reste à étudier le cas 3 divise $n$. On a, par hypothèse, $\operatorname{pgcd}(a, b, c)=1$. Donc, de $v_{2}(c)>0$, il suit que $\operatorname{pgcd}(a, b)$ est impair. On déduit alors de $v_{2}(N)>0$ et de $d \equiv 5(\bmod 8), v_{2}(N)=2$. On a donc $v_{2}(c)=v_{2}(N)-1=$ 1. Les égalités (4) impliquent alors, si 3 divise $n$,

$$
v_{2}\left(c_{n}\right)=n v_{2}(c)-v_{2}\left(f_{n}\right)=0 .
$$

D'où, d'après le tableau $1, \xi_{n}=k_{n} \sqrt{d}$.

IV. Une inégalité utile. Pour tout $n \geq 1$, soit $\left(\alpha_{i}(n)\right)_{i \geq 0}$ la suite des quotients complets du développement en fraction continue de $\alpha^{n}$. Notons $\left\langle 1, \alpha_{i}(n)\right\rangle$ le $\mathbb{Z}$-module $\mathbb{Z}+\mathbb{Z} \alpha_{i}(n)$. En utilisant l'algorithme des fractions continues, on montre que pour tout $i \geq 0$ et $n \geq 1,\left\langle 1, \alpha_{i+1}(n)\right\rangle=$ $\alpha_{i+1}(n)\left\langle 1, \alpha_{i}(n)\right\rangle$. On en déduit que pour tout $i$ et $j,\left\langle 1, \alpha_{i}(n)\right\rangle$ et $\left\langle 1, \alpha_{j}(n)\right\rangle$ sont des modules semblables et admettent donc le même anneau des stabilisateurs, $O_{n}$. Une minoration de $l\left(\alpha^{n}\right)$ en découle :

Proposition 5 (E. P. Golubeva [4]). Soient $\varphi_{n}$ l'unité fondamentale plus grande que 1 du groupe des unités de $O_{n}$ et $D_{n}$ le discriminant de $O_{n}$. Alors, pour tout $n \geq 1$,

$$
l\left(\alpha^{n}\right)>\frac{\log \varphi_{n}}{\log 2 \sqrt{D_{n}}} .
$$

Démonstration. Posons, pour tout $n \geq 1$ et $i \geq 0$,

$$
\alpha_{i}(n)=\frac{a_{i}(n)+b_{i}(n) \sqrt{d}}{c_{i}(n)}, \quad \operatorname{pgcd}\left(a_{i}(n), b_{i}(n), c_{i}(n)\right)=1 \text { et } c_{i}(n)>0 .
$$

Soit $i_{n}$ le plus petit indice $i$ tel que $\alpha_{i}(n)$ soit réduit, i.e. $\alpha_{i}(n)>1$ et $-1<\bar{\alpha}_{i}(n)<0$. On peut alors écrire, pour tout $i \geq i_{n}$,

$$
\alpha_{i}(n)<\alpha_{i}(n)-\bar{\alpha}_{i}(n)=\frac{2 b_{i}(n) \sqrt{d}}{c_{i}(n)} .
$$

Or, d'après [1], si $O$ est l'anneau des stabilisateurs du module $M=\mathbb{Z}+\mathbb{Z} \beta$, où $\beta$ non rationnel est racine du polynôme $\lambda_{1} X^{2}+\lambda_{2} X+\lambda_{3}$ avec $\left(\lambda_{1}, \lambda_{2}, \lambda_{3}\right)$ $\in \mathbb{Z}^{3}$ premiers entre eux et $\lambda_{1} \neq 0$, alors $D$, le discriminant de $O$, est égal à $D=\lambda_{2}^{2}-4 \lambda_{1} \lambda_{3}$. On en déduit $b_{i}(n)^{2} d \leq D_{n}$, et par (6), pour tout $i \geq i_{n}$,

$$
\alpha_{i}(n)<2 \sqrt{D_{n}}
$$


On a, par périodicité de la fraction continue de $\alpha^{n}$,

$$
\left\langle 1, \alpha_{i_{n}}(n)\right\rangle=\left\langle 1, \alpha_{i_{n}+l\left(\alpha^{n}\right)}(n)\right\rangle .
$$

Or

$$
\left\langle 1, \alpha_{i_{n}+l\left(\alpha^{n}\right)}(n)\right\rangle=\alpha_{i_{n}+l\left(\alpha^{n}\right)}(n) \ldots \alpha_{i_{n}+1}(n)\left\langle 1, \alpha_{i_{n}}(n)\right\rangle,
$$

et d'après la théorie des fractions continues, $\alpha_{i_{n}+l\left(\alpha^{n}\right)}(n) \ldots \alpha_{i_{n}+1}(n)=$ $\alpha_{i_{n}+l\left(\alpha^{n}\right)-1}(n) \ldots \alpha_{i_{n}}(n)$ est l'unité fondamentale strictement plus grande que 1 de l'anneau des stabilisateurs de $\left\langle 1, \alpha_{i_{n}}(n)\right\rangle$, c'est-à-dire $\varphi_{n}$. Mais $\alpha_{i}(n)$ est réduit pour tout $i \geq i_{n}$. On a alors, pour tout $n \geq 1$,

$$
\varphi_{n}=\alpha_{i_{n}+l\left(\alpha^{n}\right)}(n) \ldots \alpha_{i_{n}+1}(n)<\left(2 \sqrt{D_{n}}\right)^{l\left(\alpha^{n}\right)} .
$$

Il suit finalement

$$
l\left(\alpha^{n}\right)>\frac{\log \varphi_{n}}{\log 2 \sqrt{D_{n}}} .
$$

L'utilisation de l'inégalité (5) nécessite une majoration du discriminant $D_{n}$, qui fait l'objet du lemme suivant :

Lemme 3. Pour tout $n \geq 1, \log 2 \sqrt{D_{n}} \leq n / A$, où $A^{-1}=\log (|a|+|b| \sqrt{d})$ $+\log c+\log 4$.

Dém o n stration. En reprenant les valeurs de $\xi_{n}$ données par la proposition 2 , on a pour tout $n \geq 1, D_{n} \leq 4 c_{n}^{2} b_{n}^{2} d$. Comme $c_{n}$ est toujours inférieur ou égal à $c^{n}$, il nous suffit alors de majorer $\left|b_{n}\right|$, pour tout $n$. Considérons à nouveau les entiers $a_{n}^{\prime}$ et $b_{n}^{\prime}$ définis au paragraphe II par $(a+b \sqrt{d})^{n}=a_{n}^{\prime}+b_{n}^{\prime} \sqrt{d}$. On peut alors écrire, pour tout $n \geq 1$,

$$
\left|b_{n}\right| \sqrt{d} \leq\left|b_{n}^{\prime}\right| \sqrt{d} \leq\left|a_{n}^{\prime}\right|+\left|b_{n}^{\prime}\right| \sqrt{d}=(|a|+|b| \sqrt{d})^{n} .
$$

On en déduit

$$
\log 2 \sqrt{D_{n}} \leq \log \left(4\left|c_{n} b_{n}\right| \sqrt{d}\right) \leq n\left(\log (|a|+|b| \sqrt{d})+\log c+\frac{\log 4}{n}\right) .
$$

La minoration (5) donnée dans la proposition 5 ne devient donc effective que si l'on peut minorer de façon non triviale l'unité $\varphi_{n}$. Désignons alors par $G$ le groupe des unités de $\mathbb{Z}[\sqrt{d}]$ si $O_{n} \subset \mathbb{Z}[\sqrt{d}]$, ou de $\mathbb{Z}[(1+\sqrt{d}) / 2]$ si $O_{n} \not \subset \mathbb{Z}[\sqrt{d}]$. Si $G_{n}$ est le groupe des unités de $O_{n}$, on définit, pour tout $n \geq 1$, l'entier $\mu_{n}$ par

$$
\mu_{n}=\left[G: G_{n}\right] .
$$

Soit alors $\varepsilon_{0}$ l'unité fondamentale plus grande que 1 de $G$. Remarquons que $\varepsilon_{0}$ est l'unité fondamentale plus grande que 1 du corps $\mathbb{Q}(\sqrt{d})$, sauf si $d \equiv$ $5(\bmod 8)$ et $O_{n} \subset \mathbb{Z}[\sqrt{d}]$, où $\varepsilon_{0}$ peut éventuellement être le cube de l'unité fondamentale plus grande que 1 du corps $\mathbb{Q}(\sqrt{d})$. On a alors $\varphi_{n}=\varepsilon_{0}^{\mu_{n}}$. Nous obtenons alors par la minoration (5) et le lemme 2 l'inégalité

$$
l\left(\alpha^{n}\right)>A \frac{\mu_{n}}{n} \log \varepsilon_{0} .
$$


Notre problème se ramène donc à la détermination d'une minoration de l'indice de groupe d'unités $\mu_{n}$, ce qui fait l'objet du paragraphe suivant.

V. Etude de l'indice $\mu_{n}$. Soit, pour tout $n \geq 1, \xi_{n}$ le générateur de $O_{n}$ donné par la proposition 2. Posons $\omega=\sqrt{d}$ si $\xi_{n}=k_{n} \sqrt{d}$, et $\omega=(1+\sqrt{d}) / 2$ si $\xi_{n}=\left(1+k_{n} \sqrt{d}\right) / 2$.

Désignons, pour tout $s \geq 0$, par $P_{s} / Q_{s}$ les réduites de la fraction continue de $\omega$, et pour tout $n \geq 1$ et par $P_{s}^{(n)} / Q_{s}^{(n)}$ les réduites de la fraction continue de $\xi_{n}$. Définissons encore $\pi=l(\omega)$ et, pour tout $n \geq 1, \pi_{n}=l\left(\xi_{n}\right)$. D'après la théorie des fractions continues, on peut, pour tout $n \geq 1$, exprimer $\varphi_{n}$ à partir des réduites et de la longueur de la période de la fraction continue de $\xi_{n}$ :

$$
\varphi_{n}=P_{\pi_{n}-1}^{(n)}+Q_{\pi_{n}-1}^{(n)} \xi_{n} .
$$

De même, les puissances de $\varepsilon_{0}$ s'expriment en fonction des réduites et de la longueur de la période de la fraction continue de $\omega$ :

$$
\varepsilon_{0}^{\nu}=P_{\nu \pi-1}+Q_{\nu \pi-1} \omega, \quad \nu \geq 1 .
$$

Or comme $\varphi_{n}=\varepsilon_{0}^{\mu_{n}}$, on obtient

$$
P_{\pi_{n}-1}^{(n)}+Q_{\pi_{n}-1}^{(n)} \xi_{n}=P_{\mu_{n} \pi-1}+Q_{\mu_{n} \pi-1} \omega .
$$

En remplaçant $\xi_{n}$ et $\omega$ par leurs valeurs, il suit alors

$$
k_{n} Q_{\pi_{n}-1}^{(n)}=Q_{\mu_{n} \pi-1} .
$$

On déduit de ces égalités une minoration de l'indice $\mu_{n}=\left[G: G_{n}\right]$.

Proposition 6 (Minoration de l'indice). Soit $k_{n}=\prod_{i=1}^{s_{n}} t_{n, i}^{e_{i}(n)}$ la décomposition de $k_{n}$ en facteurs premiers, et pour tout $i=1, \ldots, s_{n}$,

$$
\begin{aligned}
& \nu_{i}(n)=\min \left\{m \geq 1: t_{n, i} \mid Q_{m \pi-1}\right\}, \\
& e_{i}^{\prime}(n)=\max \left\{e \geq 1: t_{n, i}^{e} \mid Q_{\nu_{i}(n) \pi-1}\right\}, \\
& \delta_{i}(n)= \begin{cases}0 & \text { si } e_{i}^{\prime}(n) \geq e_{i}(n), \\
e_{i}(n)-e_{i}^{\prime}(n) & \text { si } e_{i}^{\prime}(n)<e_{i}(n) .\end{cases}
\end{aligned}
$$

Alors $\mu_{n} \geq \prod_{i=1}^{s_{n}} t_{n, i}^{\delta_{i}(n)}$.

Démonstration. La preuve s'articule autour du lemme suivant :

Lemme 4 (H. Cohen [3]). Soit $\sigma$ un nombre premier. Supposons que $\sigma^{m}$ divise exactement $Q_{\gamma \pi-1}, m \geq 1, \gamma \geq 1$. Alors $\sigma^{m+1}$ divise exactement $Q_{\sigma \gamma \pi-1}$ et $\sigma^{m+1}$ ne divise pas $Q_{u \gamma \pi-1}, 1 \leq u<\sigma$.

Démonstration. D'après (8), pour tout $u \in \mathbb{N}^{*}, P_{u \gamma \pi-1}+Q_{u \gamma \pi-1} \omega$ est une unité de $G$, et $P_{u \gamma \pi-1}+Q_{u \gamma \pi-1} \omega=\left(P_{\gamma \pi-1}+Q_{\gamma \pi-1} \omega\right)^{u}$. Par la 
formule du binôme, on a alors

$$
\begin{array}{r}
Q_{u \gamma \pi-1}=\sum_{j=0}^{[(u-1) / 2]} C_{u}^{2 j+1} P_{\gamma \pi-1}^{u-2 j-1} Q_{\gamma \pi-1}^{2 j+1} d^{j} \quad \text { si } \omega=\sqrt{d} \\
\left(\text { resp. } Q_{u \gamma \pi-1}=\frac{1}{2^{u-1}} \sum_{j=0}^{[(u-1) / 2]} C_{u}^{2 j+1}\left(2 P_{\gamma \pi-1}-Q_{\gamma \pi-1}\right)^{u-2 j-1} Q_{\gamma \pi-1}^{2 j+1} d^{j},\right. \\
\text { si } \left.\omega=\frac{1+\sqrt{d}}{2}\right) .
\end{array}
$$

Or par hypothèse, $\sigma^{m}$ divise exactement $Q_{\gamma \pi-1}$. Alors $\sigma^{2 m}$ divise tous les membres de la somme, sauf peut être

$$
\begin{gathered}
C_{u}^{1} P_{\gamma \pi-1}^{u-1} Q_{\gamma \pi-1}=u P_{\gamma \pi-1}^{u-1} Q_{\gamma \pi-1} \quad \text { si } \omega=\sqrt{d} \\
\left(\text { resp. } \frac{C_{u}^{1}}{2^{u-1}}\left(2 P_{\gamma \pi-1}-Q_{\gamma \pi-1}\right)^{u-1} Q_{\gamma \pi-1}\right. \\
\left.\quad=\frac{u}{2^{u-1}}\left(2 P_{\gamma \pi-1}-Q_{\gamma \pi-1}\right)^{u-1} Q_{\gamma \pi-1}\right) .
\end{gathered}
$$

Si $\sigma \neq 2$, alors $\sigma$ ne divise pas $P_{\gamma \pi-1}$ car sinon, comme $P_{\gamma \pi-1}^{2}-Q_{\gamma \pi-1}^{2} d=$ \pm 1 (resp. $\left(2 P_{\gamma \pi-1}-Q_{\gamma \pi-1}\right)^{2}-Q_{\gamma \pi-1}^{2} d= \pm 4$ ), alors $\sigma$ divise 1 (resp. $\sigma$ divise 4 ), ce qui est absurde. On voit alors que $\sigma$ est le plus petit entier $u$ tel que $\sigma^{m+1}$ divise $u P_{\gamma \pi-1}^{u-1} Q_{\gamma \pi-1}$ (resp. $\sigma^{m+1}$ divise $\frac{u}{2^{u-1}}\left(2 P_{\gamma \pi-1}-\right.$ $\left.\left.Q_{\gamma \pi-1}\right)^{u-1} Q_{\gamma \pi-1}\right) . \sigma$ est alors le plus petit $u$ tel que $\sigma^{m+1}$ divise $Q_{u \gamma \pi-1}$, et de plus, $\sigma^{m+1}$ divise exactement $Q_{\sigma \gamma \pi-1}$.

Montrons que si $\sigma=2$, alors $\omega=\sqrt{d}$. Raisonnons par l'absurde, et supposons $\omega=(1+\sqrt{d}) / 2$. On a alors $d \equiv 1(\bmod 4)$, et 2 divise $2 P_{\gamma \pi-1}-$ $Q_{\gamma \pi-1}$ si $m>1$ ou 4 divise $2 P_{\gamma \pi-1}-Q_{\gamma \pi-1}$ si $m=1$. D'où, $\left(2 P_{\gamma \pi-1}-\right.$ $\left.Q_{\gamma \pi-1}\right)^{2}-Q_{\gamma \pi-1}^{2} d$ est impair, ce qui est absurde.

Donc si $\sigma=2$ alors $\omega=\sqrt{d}$, et avec les mêmes arguments que précédemment, 2 est le plus petit entier $u$ tel que $2^{m+1}$ divise $Q_{u \gamma \pi-1}$ et $2^{m+1}$ divise exactement $Q_{2 \gamma \pi-1}$.

Désignons alors par $\mu_{i}(n), i=1, \ldots, s_{n}$, le plus petit entier positif $m$ tel que $t_{n, i}^{e_{i}(n)}$ divise $Q_{m \pi-1}$, son existence étant assurée par (9). En reprenant la démonstration du lemme 4 en remplaçant $\sigma^{m}$ par $t_{n, i}^{e_{i}(n)}$, on voit que pour tout $u \geq 1, t_{n, i}^{e_{i}(n)}$ divise $Q_{u \mu_{i}(n) \pi-1}$. Donc si $\mu_{n}^{\prime}$ est le plus petit entier positif $m$ tel que $k_{n}$ divise $Q_{m \pi-1}$, on a $\mu_{n}^{\prime}=\operatorname{ppcm}\left(\mu_{i}(n)\right)$. Or, $t_{n, i}^{e_{i}^{\prime}(n)}$ divise exactement $Q_{\nu_{i}(n) \pi-1}$. On en déduit donc, grâce au lemme $4, \mu_{i}(n)=\nu_{i}(n) t_{n, i}^{\delta_{i}(n)}$. 
Et comme les $t_{n, i}$ sont premiers entre eux,

$$
\mu_{n}^{\prime}=\operatorname{ppcm}\left(\nu_{i}(n)\right) \prod_{i=1}^{s_{n}} t_{n, i}^{\delta_{i}(n)}
$$

c'est-à-dire,

$$
\mu_{n}^{\prime} \geq \prod_{i=1}^{s_{n}} t_{n, i}^{\delta_{i}(n)}
$$

Mais par la définition de $\mu_{n}^{\prime}$ et (8), $\varepsilon_{0}^{\mu_{n}^{\prime}}$ est une unité de $O_{n}$. Comme $\varepsilon_{0}^{\mu_{n}}$ est l'unité fondamentale de $O_{n}$, il suit alors que $\mu_{n}$ divise $\mu_{n}^{\prime}$. Or, par (9), $\mu_{n}^{\prime} \leq$ $\mu_{n}$, d'où $\mu_{n}=\mu_{n}^{\prime}$, ce qui termine la démonstration de la proposition 6 .

On en déduit le corollaire suivant :

Corollaire. Si $\lambda$ est un entier plus grand que 1 et si $\lambda^{m}$ divise $k_{n}$, il existe un entier $r$ indépendant de $m$ tel que $\mu_{n} \geq \lambda^{m-r}$.

VI. Minoration de $l\left(\alpha^{n}\right)$. Il apparaît que la minoration donnée par le corollaire de la proposition 6 est intéressante si $k_{n}$ est divisible par une puissance élevée d'un nombre entier. L'ensemble des nombres réels quadratiques pour lesquels l'application de ce résultat permet de conclure est donné par le théorème suivant.

ThÉORÈme. Soit $\alpha=(a+b \sqrt{d}) / c, a \in \mathbb{Z}, b \in \mathbb{Z}^{*}, c \in \mathbb{N}^{*}, \operatorname{pgcd}(a, b, c)=$ 1 et $d \geq 2$ sans facteur carré. Désignons par $\varepsilon_{0}$ l'unité fondamentale plus grande que 1 du corps $\mathbb{Q}(\sqrt{d})$ ou son cube. Posons $A^{-1}=\log (|a|+|b| \sqrt{d})+$ $\log c+\log 4$ et $\varrho=\operatorname{pgcd}(a, d, c)$. Supposons que $\alpha$ vérifie au moins l'une des conditions suivantes :

(i) $N_{2} \not \equiv 0\left(\bmod c_{2}\right)$;

(ii) $\operatorname{pgcd}(a, b)>1$;

(iii) $\operatorname{pgcd}(a, d)>1$;

(iv) $a^{2}-b^{2} d$ pair et c impair.

Alors il existe une constante effectivement calculable $r$ ne dépendant que de $\alpha$ et deux entiers $\lambda \geq 2$ et $f(n) \geq[n / 2]$ tels que pour tout $n \geq 2$,

$$
l\left(\alpha^{n}\right) \geq A \log \varepsilon_{0} \frac{\lambda^{f(n)-r}}{n},
$$

où l'on peut prendre comme valeurs de $\lambda$ et $f(n)$ :

- si a vérifie (i), celles données par le tableau 2;

- si $\alpha$ vérifie (ii), $\lambda=\operatorname{pgcd}(a, b)$ et $f(n)=n$;

- si $\alpha$ vérifie (iii), $\lambda=\operatorname{pgcd}(a, d)$ et $f(n)=[n / 2]$;

- si $\alpha$ vérifie (iv), $\lambda=2$ et $f(n)=v_{2}\left(\delta_{n}\right)$ donné par le lemme 2 . 
Tableu 2

\begin{tabular}{|c|c|c|c|}
\hline & $\begin{array}{c}\xi_{n}=k_{n} \sqrt{d} \\
\text { et } \\
c / \gamma_{1} \text { entier }\end{array}$ & $\begin{array}{c}\xi_{n}=k_{n} \sqrt{d} \\
\text { et } \\
c / \gamma_{1} \text { non entier }\end{array}$ & $\xi_{n}=\left(1+k_{n} \sqrt{d}\right) / 2$ \\
\hline$N \not \equiv 0(\bmod c)$ & $\begin{array}{c}\lambda=\left(c / \gamma_{1}\right), f(n)=n \\
\quad \text { avec } c / \gamma_{1}>1\end{array}$ & $\begin{array}{c}\lambda=\left(2 c / \gamma_{1}\right), f(n)=n \\
\text { avec } 2 c / \gamma_{1}>1\end{array}$ & $\begin{array}{c}\lambda=\left(2 c / \gamma_{1}\right), f(n)=n \\
\text { avec } 2 c / \gamma_{1}>1\end{array}$ \\
\hline $\begin{array}{c}N \equiv 0(\bmod c) \\
\text { et } \\
v_{2}(c)<v_{2}(N) \\
\text { ou } v_{2}(c)=0\end{array}$ & $\begin{array}{c}\lambda=\varrho, \quad f(n)=[n / 2] \\
\text { avec } \varrho>1\end{array}$ & $\begin{array}{c}\lambda=\varrho, f(n)=[n / 2] \\
\text { avec } \varrho>1\end{array}$ & $\begin{array}{c}\lambda=\varrho, f(n)=[n / 2] \\
\text { avec } \varrho>1\end{array}$ \\
\hline $\begin{array}{c}N \equiv 0(\bmod c) \\
\text { et } \\
v_{2}(c)=v_{2}(N)>0\end{array}$ & $\lambda=2$ et $f(n)=l_{n}^{\prime}$ & impossible & impossible \\
\hline
\end{tabular}

Nous justifions dans la remarque suivante les situations impossibles du tableau 2.

Remarque. Par hypothèse, pour tout $n \geq 1, \operatorname{pgcd}\left(a_{n}, b_{n}, c_{n}\right)=1$, et les entiers $\gamma_{n}$ et $b_{n}$ n'ont pas de facteur commun. On en déduit donc, puisque par définition $k_{1}$ est entier, que si $c / \gamma_{1}$ n'est pas entier, alors $k_{1}=\left|2 c b / \gamma_{1}\right|$, et par la proposition $2, \xi_{1}=\left(1+k_{1} \sqrt{d}\right) / 2$. D'où, d'après le tableau 1 , $0<v_{2}(c)<v_{2}(N)$.

Par conséquent, avoir $c / \gamma_{1}$ non entier et $v_{2}(c) \geq v_{2}(N)$ est impossible. De même, si $\xi_{n}=\left(1+k_{n} \sqrt{d}\right) / 2$, grâce à la proposition 4 , on a $\xi_{1}=\left(1+k_{1} \sqrt{d}\right) / 2$, et par suite $0<v_{2}(c)<v_{2}(N)$.

Afin de dégager des puissances élevées de nombres entiers dans les diviseurs de $k_{n}$, nous allons, dans le lemme 6 , donner une factorisation de l'entier $k_{n}$. D'après la proposition 2 , on a $k_{n}=\left|c_{n} b_{n} / \gamma_{n}\right|$ avec $c_{n} / \gamma_{n} \in \mathbb{N}^{*}$ si $\xi_{n}=k_{n} \sqrt{d}$, et $k_{n}=\left|2 c_{n} b_{n} / \gamma_{n}\right|$ avec $2 c_{n} / \gamma_{n} \in \mathbb{N}^{*}$ si $\xi_{n}=\left(1+k_{n} \sqrt{d}\right) / 2$. Il est alors nécessaire de déterminer l'entier $\gamma_{n}$ en fonction de $\alpha$, ce qui fait l'objet du lemme 5 .

Lemme 5. Si pour tout $n \geq 1 \gamma_{n}=\operatorname{pgcd}\left(2 c_{n}, a_{n}^{2}-b_{n}^{2} d, c_{n}^{2}\right)$, alors $\gamma_{1}^{n}=$ $\gamma_{n} f_{n}^{2}$.

Démonstration. Soit, pour tout $n \geq 1, \omega_{1}(n) X^{2}+\omega_{2}(n) X+\omega_{3}(n)$ $=0$ l'équation minimale de $\alpha^{n}$ introduite au paragraphe III. Posons, pour alléger l'écriture, $\omega_{1}=\omega_{1}(1), \omega_{2}=\omega_{2}(1)$ et $\omega_{3}=\omega_{3}(1)$. On montre par récurrence sur $n$ que l'équation minimale de $\alpha^{n}$ est $\omega_{1}^{n} X^{2}+\omega_{2}(n) X+\omega_{3}^{n}=0$.

De $\left(\omega_{1} \alpha^{2}+\omega_{3}\right)^{2}=\omega_{2}^{2} \alpha^{2}$, on obtient $\omega_{1}^{2} \alpha^{4}+\left(2 \omega_{1} \omega_{3}-\omega_{2}^{2}\right) \alpha^{2}+\omega_{3}^{2}=0$, ce qui montre la propriété pour $n=2$. Supposons la propriété vraie jusqu'au 
rang $n$. On peut écrire

$$
\left(\omega_{1}^{n} \alpha^{2 n}+\omega_{3}^{n}\right)\left(\omega_{1} \alpha^{2}+\omega_{2} \alpha+\omega_{3}\right)=0 .
$$

En développant, on obtient

$$
\omega_{1}^{n+1} \alpha^{2 n+2}+\omega_{3}^{n+1}+\left(\omega_{1}^{n} \omega_{2} \alpha^{2 n+1}+\omega_{1}^{n} \omega_{3} \alpha^{2 n}+\omega_{3}^{n} \omega_{1} \alpha^{2}+\omega_{3}^{n} \omega_{2} \alpha\right)=0 .
$$

Or,

$$
\omega_{1}^{n} \omega_{2} \alpha^{2 n+1}+\omega_{3}^{n} \omega_{2} \alpha=-\omega_{2} \omega_{2}(n) \alpha^{n+1}
$$

et

$$
\omega_{1}^{n} \omega_{3} \alpha^{2 n}+\omega_{3}^{n} \omega_{1} \alpha^{2}=-\omega_{3} \omega_{1} \omega_{2}(n-1) \alpha^{n+1} .
$$

On en déduit alors

$$
\omega_{1}^{n+1} \alpha^{2 n+2}-\left(\omega_{1} \omega_{3} \omega_{2}(n-1)+\omega_{2} \omega_{2}(n)\right) \alpha^{n+1}+\omega_{3}^{n+1}=0 .
$$

Si $p>1$ premier divise $\operatorname{pgcd}\left(\omega_{1}, \omega_{3}\right)$, alors il ne divise le coefficient de $\alpha^{n+1}$ que s'il divise $\omega_{2}$, car d'après l'hypothèse de récurrence $p$ ne divise pas $\omega_{2}(n)$. Or $\operatorname{pgcd}\left(\omega_{1}, \omega_{2}, \omega_{3}\right)=1$. La propriété est donc vérifiée au rang $n+1$.

D'après (3), on a pour tout $n \geq 1, \omega_{1}(n)=c_{n}^{2} / \gamma_{n}$. Il en suit

$$
\omega_{1}(n)=\omega_{1}^{n}=\frac{c_{n}^{2}}{\gamma_{n}}=\left(\frac{c^{2}}{\gamma_{1}}\right)^{n} .
$$

De $c^{n}=c_{n} f_{n}$, on déduit alors $\gamma_{1}^{n}=\gamma_{n} f_{n}^{2}$.

Lemme 6. Posons $\varrho=\operatorname{pgcd}(a, d, c)$. Alors pour tout $n \geq 1$, une factorisation de $k_{n}$ est donnée par le tableau suivant:

Tableau 3

\begin{tabular}{|c|c|c|c|}
\hline & $\begin{array}{c}\xi_{n}=k_{n} \sqrt{d} \\
\text { et } \\
c / \gamma_{1} \text { entier }\end{array}$ & $\begin{array}{c}\xi_{n}=k_{n} \sqrt{d} \\
\text { et } \\
c / \gamma_{1} \text { non entier }\end{array}$ & \\
& $\xi_{n}=\left(1+k_{n} \sqrt{d}\right) / 2$ \\
\hline$k_{n}$ & $\left(c / \gamma_{1}\right)^{n} \varrho^{[n / 2]} 2^{l_{n}^{\prime}}\left|b_{n}\right|$ & $\left(2 c / \gamma_{1}\right)^{n} \varrho^{[n / 2]}\left|b_{n}\right|$ & $\left(2 c / \gamma_{1}\right)^{n} \varrho^{[n / 2]}\left|b_{n}\right|$ \\
\hline
\end{tabular}

Démonstration. Si $\xi_{n}=k_{n} \sqrt{d}$, alors d'après la proposition $2, k_{n}=$ $\left|c_{n} b_{n} / \gamma_{n}\right|$, et si de plus $c / \gamma_{1}$ est entier, de l'égalité $c^{n}=c_{n} f_{n}$ et du lemme 4 , on écrit

$$
k_{n}=\left(c / \gamma_{1}\right)^{n} f_{n}\left|b_{n}\right| .
$$

Et, d'après la proposition 1 , on a $f_{n}=2^{l_{n}^{\prime}} \varrho^{[n / 2]}$.

Si $\xi_{n}=k_{n} \sqrt{d}$, alors $k_{n}=\left|c_{n} b_{n} / \gamma_{n}\right|$, et si $c / \gamma_{1}$ n'est pas un entier, alors par définition de $\gamma_{n}, 2 c / \gamma_{1}$ est un entier. D'où, grâce au lemme 4 ,

$$
k_{n}=\left(2 c / \gamma_{1}\right)^{n}\left(f_{n} / 2^{n}\right)\left|b_{n}\right|=\left(2 c / \gamma_{1}\right)^{n} \varrho^{[n / 2]} 2^{l_{n}^{\prime}-n}\left|b_{n}\right| .
$$

Mais de $c / \gamma_{1}$ non entier, on déduit, comme dans la remarque du tableau 2, $\xi_{1}=\left(1+k_{1} \sqrt{d}\right) / 2$. Il suit, par le tableau $1, v_{2}(N)>v_{2}(c)>0$. De plus, 
comme $\xi_{n}=k_{n} \sqrt{d}$, on obtient, grâce à la proposition $4, d \equiv 5(\bmod 8)$ et 3 divise $n$, d'où finalement, d'après la proposition $1, l_{n}^{\prime}=n$.

Si $\xi_{n}=\left(1+k_{n} \sqrt{d}\right) / 2$, alors $k_{n}=\left|2 c_{n} b_{n} / \gamma_{n}\right|$. Par définition, $2 c / \gamma_{1}$ est un entier, et on écrit grâce au lemme 4 ,

$$
k_{n}=\left(2 c / \gamma_{1}\right)^{n} f_{n}\left|b_{n}\right|=\left(2 c / \gamma_{1}\right)^{n} \varrho^{[n / 2]} 2^{l_{n}^{\prime}-(n-1)}\left|b_{n}\right| .
$$

De la proposition 4 , on déduit $\xi_{1}=\left(1+k_{1} \sqrt{d}\right) / 2$ et $d \equiv 1(\bmod 8)$ ou $d \equiv$ $5(\bmod 8)$ avec $n$ non divisible par 3 . Du tableau 1 , on tire alors $v_{2}(N)>$ $v_{2}(c)>0$, et par suite, d'après la proposition $1, l_{n}^{\prime}=n-1$.

Démonstration du théorème. Nous allons, pour chacune des quatre conditions du théorème, déterminer $\lambda \geq 2$ et $f(n) \geq[n / 2]$ tels que pour tout $n \geq 2, \lambda^{f(n)}$ divise $k_{n}$. Par suite, grâce à l'inégalité $(7)$ et au corollaire de la proposition 6 , on déduit qu'il existe un entier $r$ indépendant de $f(n)$ tel que pour tout $n \geq 2$,

$$
l\left(\alpha^{n}\right)>A \log \varepsilon_{0} \frac{\lambda^{f(n)}}{n} \geq A \log \varepsilon_{0} \frac{2^{[n / 2]}}{n} .
$$

(i) $N_{2} \not \equiv 0\left(\bmod c_{2}\right)$. Fixons $n \geq 2$. Comme $c_{2}$ ne divise pas $N_{2}$, en prenant la contraposée de la proposition 3(i), pour tout $n \geq 2, c_{n}$ ne divise pas $N_{n}$. D'où par la proposition 2(ii), $k_{n}>\left|b_{n}\right|$. C'est-à-dire, d'après le lemme 6 :

$$
\frac{k_{n}}{\left|b_{n}\right|}=\left\{\begin{array}{l}
\left(\frac{c}{\gamma_{1}}\right)^{n} \varrho^{[n / 2]} 2^{l_{n}^{\prime}}>1 \\
\text { ou } \\
\left(\frac{2 c}{\gamma_{1}}\right)^{n} \varrho^{[n / 2]}>1 .
\end{array}\right.
$$

Or, grâce à la proposition 3(ii), $c_{2}$ ne divise pas $N_{2}$ est équivalent à : $c$ ne divise pas $N$, ou $\varrho=\operatorname{pgcd}(a, d, c)>1$, ou $v_{2}(c)=v_{2}(N)>0$. Notons que la dernière condition devrait être $v_{2}(c) \geq v_{2}(N)>0$. Mais $v_{2}(c)=v_{2}(N)>0$ suffit, car si $v_{2}(c)>v_{2}(N)$ alors $c$ ne divise pas $N$.

Si $c$ ne divise pas $N$, alors d'après la proposition $2(\mathrm{ii}), c / \gamma_{1}>1$ ou $2 c / \gamma_{1}>1$. On en déduit donc par (10) que pour tout $n \geq 1,\left(c / \gamma_{1}\right)^{n}$ divise $k_{n}$ si $\xi_{n}=k_{n} \sqrt{d}$ et $c / \gamma_{1}$ est entier, ou $\left(2 c / \gamma_{1}\right)^{n}$ divise $k_{n}$ si $\xi_{n} \neq k_{n} \sqrt{d}$ ou si $c / \gamma_{1}$ n'est pas entier.

Si $c$ divise $N$ et $v_{2}(c)<v_{2}(N)$ ou $v_{2}(c)=0$, alors $\varrho=\operatorname{pgcd}(a, d, c)>1$, alors par (10), pour tout $n \geq 1, \varrho^{[n / 2]}$ divise $k_{n}$.

Si $c$ divise $N$ et $v_{2}(c)=v_{2}(N)>0$, alors d'après la proposition 1 , pour $d \not \equiv 2(\bmod 4)$ et pour tout $n \geq 1, l_{n}^{\prime} \geq[n / 2]$. Donc par $(10)$, si $\xi_{n}=k_{n} \sqrt{d}$ et $c / \gamma_{1}$ est entier, $2^{[n / 2]}$ divise $k_{n}$. Si $d \equiv 2(\bmod 4)$, alors forcément 2 divise $\operatorname{pgcd}(a, d, c)$ et on revient au cas précédent.

(ii) $\operatorname{pgcd}(a, b)>1$. Comme $\operatorname{pgcd}(a, b, c)=1$, il suit que pour tout $n \geq 1$, $(\operatorname{pgcd}(a, b))^{n}$ divise $b_{n}$, et donc divise $k_{n}$. 
(iii) $\operatorname{pgcd}(a, d)>1$. Si $\varrho=\operatorname{pgcd}(a, d, c)$, alors d'après la proposition 1 , pour tout $n \geq 1, \varrho^{[n / 2]}$ divise $f_{n}$. D'où $(\operatorname{pgcd}(a, d))^{[n / 2]}$ divise $\delta_{n}=$ $\operatorname{pgcd}\left(a_{n}^{\prime}, b_{n}^{\prime}\right)$, et donc $(\operatorname{pgcd}(a, d) / \varrho)^{[n / 2]}$ divise $b_{n}$. Or, si $\varrho>1$, en revenant à la démonstration du (i), $\varrho^{[n / 2]}$ divise $k_{n}$. Par conséquent, $(\operatorname{pgcd}(a, d))^{[n / 2]}$ divise $k_{n}$.

(iv) $N=a^{2}-b^{2} d$ pair et $c$ impair. Pour tout $n \geq 1, b_{n}$, et donc $k_{n}$, est divisible par $2^{v_{2}\left(\delta_{n}\right)}$, avec $v_{2}\left(\delta_{n}\right)$ donné par le lemme 2 .

\section{Références}

[1] Z. I. Borevitch et I. R. Chafarevitch, Théorie des nombres, Gauthier-Villars, 1967.

[2] G. Choquet, Répartition des nombres $k(3 / 2)^{n}$; et ensembles associés; Algorithmes adaptés aux suites $\left(k \theta^{n}\right)$ et aux chaînes associées; $\theta$-jeux récursifs et application aux suites $\left(k \theta^{n}\right)$; solenö̈des de $T^{z}$, C. R. Acad. Sci. Paris Sér. A 290 (1980), 575-580; 719-724 et 863-868; Construction effective des suites $k(3 / 2)^{n}$. Etude des mesures $3 / 2$-stables de $\Pi$; Les fermés $(3 / 2)$-stables de $T$; structure des fermés dénombrables; applications arithmétiques, ibid. 291 (1980), 69-74 et 239-244; $\theta$-fermés, $\theta$-chaînes et $\theta$-cycles (pour $\theta=3 / 2$ ); $\theta$-fermés et dimension de Hausdorff. Conjectures de travail. Arithmétique des $\theta$-cycles ( où $\theta=3 / 2$ ), C. R. Acad. Sci. Paris Sér. I 292 (1981), 5-10 et 339-344.

[3] H. Cohen, Multiplication par un entier d'une fraction continue périodique, Acta Arith. 26 (1974), 129-148.

[4] E. P. Golubeva, On the length of the period of a quadratic irrationality, Math. USSR-Sb. 51 (1) (1985), 119-129.

[5] M. Mendès France, Remarks on finite continued fractions, Enseign. Math. 39 (1993), 249-257.

[6] R. Paysant-Le Roux et E. Dubois, Une application des nombres de Pisot à l'algorithme de Jacobi-Perron, Monatsh. Math. 98 (1984), 145-155.

[7] A. J. van der Poorten, Some problems of recurrent interest, in: Topics in Classical Number Theory (Colloq. Budapest, 1981), Colloq. Math. Soc. János Bolyai 34, G. Halász (éd.), Vol. 2, North-Holland, 1984, 1265-1294.

DÉPARTEMENT DE MATHÉMATIQUES

UNIVERSITÉ DE CAEN

ESPLANADE DE LA PAIX

14032 CAEN CEDEX, FRANCE

E-mail: GRISEL@MATH.UNICAEN.FR 